Revue internationale P.M.E.

Économie et gestion de la petite et moyenne entreprise

\title{
Crise, politique régionale et PME en Hongrie
}

\section{Gyula Horváth}

Volume 5, numéro 1, 1992

URI : https://id.erudit.org/iderudit/1008130ar

DOI : https://doi.org/10.7202/1008130ar

Aller au sommaire du numéro

Éditeur(s)

Presses de l’Université du Québec

ISSN

0776-5436 (imprimé)

1918-9699 (numérique)

Découvrir la revue

Citer cet article

Horváth, G. (1992). Crise, politique régionale et PME en Hongrie. Revue internationale P.M.E., 5(1), 9-26. https://doi.org/10.7202/1008130ar

\section{Résumé de l'article}

Le passage à une économie moderne, en favorisant la transformation de la structure économique, est nécessaire pour que la Hongrie puisse sortir de la crise actuelle. L'objet de cet article est de présenter le contexte dans lequel révolution rapide du secteur des PME pousse l'économie hongroise vers la décentralisation économique, régionale et politique. L’auteur présente les dimensions régionales de la crise en décrivant les principaux éléments qui ont exercé une influence sur cette crise ainsi que le contexte économique général qui rend difficile la réorganisation de cette économie.

Après un rappel historique des circonstances qui ont amené la formation du système des entreprises, l'auteur présente les différentes législations adoptées par l'État illustrant comment l'économie hongroise a changé rapidement son système politique et économique avec une structure industrielle où les PME sont devenues très importantes. Enfin, l'auteur explique comment la localisation et l'expansion territoriale des entreprises s'effectue de façon inégale d'une région à l'autre. 


\title{
Crise, politique régionale et PME en Hongrie
}

\author{
Gyula HORVÁTH \\ Institut des Sciences de Transdanubie à Pécs
}

\begin{abstract}
RÉSUMÉ
Le passage à une économie moderne, en favorisant la transformation de la structure économique, est nécessaire pour que la Hongrie puisse sortir de la crise actuelle. L'objet de cet article est de présenter le contexte dans lequel l'évolution rapide du secteur des PME pousse l'économie hongroise vers la décentralisation économique, régionale et politique. L'auteur présente les dimensions régionales de la crise en décrivant les principaux éléments qui ont exercé une influence sur cette crise ainsi que le contexte économique général qui rend difficile la réorganisation de cette économie.
\end{abstract}

Après un rappel historique des circonstances qui ont amené la formation du système des entreprises, l'auteur présente les différentes législations adoptées par l'État illustrant comment l'économie hongroise a changé rapidement son système politique et économique avec une structure industrielle où les PME sont devenues très importantes. Enfin, l'auteur explique comment la localisation et l'expansion territoriale des entreprises s'effectue de façon inégale d'une région à l'autre.

\section{ABSTRACT}

The transition to a modern economy which promotes the transformation of the economic structure is necessary for Hungary to overcome its present crisis. The object of this article is to present the context in which the rapid evolution of the SMB sector is forcing the Hungarian economy toward an economic decentralization, both regional and political. The author presents the regional dimensions of the crisis by describing the principal elements which have influenced this crisis as well as the general economic context which makes economic reorganization difficult.

Following a historical summary of the circumstances which lead to the formation of the entrepreneurial system, the author presents the different laws adopted by the state thus showing how the Hungarian economy rapidly changed its political and economic system to an industrial structure in which SMBs have become very important. Finally, the author explains how the localisation and territorial expansion of businesses is taking place in a way which is not equal from one region to the other. 


\section{RESUMEN}

La transición a una economía moderna permitiendo la transformación de la estructura económica es necesaria para que Hungría salga de la crisis actual. El objectivo de éste artículo es de presentar el contexto dentro del cual la evolucion rápida del sector de las PME empuja la economía hungara hacia la decentralización económica, regional y política. El autor presenta las dimensiónes regionales de la crisis describiendo los principales elementos que han ejercido una influencia sobre ésta crisis y tambien que el contexto económico general que hace dificil la reorganizacion de esta economía.

Despues de un recuerdo histórico de las circunstancias que han dirigido la formacion del sistema de las empresas, el autor presenta las diferentes legislaciones adoptadas por el Estado ilustrando como la economía hungara a cambio rápidamente su sistema politico y económico por una estructura industrial donde las PME son devenidas muy importantes. Enfin, el autor explica como la localizacion y la expansion territorial de las empresas se effectuan de una manera inigual de una region a la otra.

\section{Les dimensions régionales de la crise}

La crise économique, dans une société hongroise également en crise, fait sentir son influence dans tous les domaines. Elle modifie et oblige à revaloriser les données du développement. Les conséquences de cette crise diffèrent toutefois selon les structures qu'elle affecte. Dans les régions à structures faibles ou dans les agglomérations à monoculture, la stagnation ou la régression économiques font apparaître de nouveaux phénomènes régionaux, comme le chômage et l'appauvrissement croissant. Avec les nombreux problèmes de développement que connaissent plusieurs branches d'activités et de nombreuses entreprises dans les régions plus industrialisées, on voit poindre un processus présentant les signes d'une décadence socio-économique certaine. L'aggravation des conditions économiques du secteur agraire entraîne à la stagnation des régions agricoles. La capacité de production de revenu des branches les plus productives diminue peu à peu ; ces activités perdent graduellement leurs moyens pour soutenir le développement des régions et des agglomérations. Faute de ressources et d'une économie politique cohérente, les programmes de développement pour les régions en retard sont très peu efficaces.

Ainsi, le développement des régions hongroises les plus problématiques (où la crise est plus aiguë) arrive à un tournant décisif. On ne peut plus aller de l'avant et favoriser l'aménagement du territoire comme on l'a fait jusqu'à présent. Il faut passer à une économie moderne, c'est-à-dire favoriser la transformation de la structure économique pour sortir de la crise. 
La politique de l'aménagement du territoire des décennies précédentes en Hongrie, s'était fixé comme premier objectif la diminution des grandes inégalités. Cette politique a permis de déterminer, à plusieurs reprises et de façons différentes, le seuil d'inégalité au-delà duquel il était considéré comme non souhaitable, irraisonnable et politiquement inique, en insistant sur sa diminution à plus ou moins long terme, sur sa liquidation progressive. Il demeure que le territoire concerné par cet objectif de diminution des inégalités a varié à plusieurs reprises. Cette politique a tantôt visé les différences entre les départements à l'intérieur des régions et tantôt les différences de développement entre la capitale et les provinces, les villages et les villes, les régions industrielles et les régions agricoles.

À partir de résultats partiels et provisoires, on peut affirmer que ces efforts ne furent pas en général couronnés de succès. Au contraire, l'aggravation de l'environnement socio-économique, l'expansion de la crise forçant un changement de structure économique et la transformation du système politique n'ont fait qu'augmenter les inégalités, et l'image d'une forte polarisation s'est confirmée.

En effet, en Hongrie quatre éléments - chacun d'entre eux accentuant la crise régionale - ont exercé simultanément leur influence :

- le problème des régions désavantagées, désavantage accumulé au cours de l'histoire, le retard croissant des régions rurales et l'impossibilité de développer les régions agricoles sans pôle de développement ;

- le changement de rôle et des rapports du marché dans certains domaines d'activités, surtout dans le cas où ces domaines jouent un rôle décisif dans la structure d'une région, alors qu'un changement radical de structure économique régionale devient nécessaire. L'exemple typique est le cas des régions où l'industrie lourde domine ou des régions traditionnellement dépressives ;

- l'effondrement rapide du système économique de l'Europe de l'Est et la nécessité de s'orienter vers la nouvelle économie mondiale et le système de marché, qui met l'économie entière dans un état critique, mais de façons différentes selon les régions ;

- à la suite du changement du système socio-économique, la transformation de la structure d'organisation et de fonctionnement de l'économie, avec, d'une part, le changement de rapports de propriété (la privatisation) et d'autre part, la transformation du système d'organisation économique ou la décomposition sélective du système des grandes entreprises auparavant déterminant et la création rapide de nombreuses PME. 
Le chômage constitue la conséquence la plus évidente de la crise régionale dont la première cause est la désintégration du secteur public. Avec la répartition territoriale de l'industrie lourde publique ainsi que l'inégalité régionale du développement de la structure économique, le chômage entraîne de graves tensions sociales surtout dans les régions de l'Est du pays (dans les régions rurales de la Planie Hongroise, industrialisées dans les années 60 mais qui, avec une technologie périmée, ont eu à subir le transfert des usines vers Budapest) et au centre, où l'économie repose sur l'industrie lourde et d'exploitation (à Miskolc et aux alentours). En novembre 1991, le taux de chômage dans le pays atteignait 7,3\% avec 351000 chômeurs ; dans les régions de l'Est du pays, il variait entre $10 \%$ et $14 \%$; dans celles de l'Ouest, il était entre $4 \%$ et $7 \%$; et à Budapest, il n'y avait que $2,4 \%$ de chômeurs. L'accroissement rapide du taux de chômage peut être démontré par le fait qu'en janvier 1990, il y avait 23000 chômeurs recensés ; en janvier 1991, il y en avait 100000 et en juin, 186000. On voit que le nombre de chômeurs entre le début de l'année 1990 et la fin de 1991, s'est multiplié par 17.

La crise générale du secteur d'État a sapé les bases du marché de la maind'œuvre publique en Hongrie. Actuellement, avec la diminution de la production et la chute des effectifs dans l'industrie étatique (et de l'expansion des PME dont on parlera plus loin) on peut voir se profiler à l'horizon les contours de trois types de régions. Les régions centrales (Budapest et les départements voisins) sont caractérisées par le retrait de l'industrie publique et le développement rapide de l'économie privée. Les régions de l'Est, de la Plaine et du Nord-Est sont caractérisées par une profonde dépression. Les autres régions du pays sont en transition, leur appartenance à un des types que nous venons de mentionner n'est pas encore établie.

En Europe centrale-orientale, trois changements fondamentaux hérités de l'histoire se sont conjugués au cours des 15 dernières années (Gy Enyedi, 1990). Premièrement, avec l'achèvement de l'industrialisation, on a dû passer à l'étape postindustrielle qui s'est déroulée en Europe de l'Ouest et dans l'économie mondiale des années 60 . Deuxièmement, on a assisté au changement de structure et de technologie au regard duquel l'économie hongroise s'est montrée complètement inapte. Troisièmement, on a dû faire face au changement du système sociopolitique, car le modèle socioéconomique du socialisme a étouffé l'évolution des forces productrices.

La réorganisation de l'économie est rendue difficile par le fait qu'il faut répondre à tous ces défis en même temps : il faut réaliser la modernisation in-

1. Ces données se rapportent au premier trimestre. 
terne technologique-technique, la réorganisation de l'industrie hongroise et la restructuration de l'économie générale. On doit réaliser tout cela à une époque où :

- le taux de croissance de l'économie diminue et son déclin semble inéluctable ;

- l'efficacité est à peu de choses près inexistante, les ressources de l'économie sont gaspillées ;

- une grande partie de la population s'appauvrit, la polarité de la société dépasse le niveau qui est propre en général aux économies de marché ;

- l'inflation s'accélère ;

- le chômage et la dette extérieure et intérieure sont en hausse ;

- les inégalités régionales s'accroissent très rapidement.

Malgré tout, de nouvelles idées pour la modernisation de l'économie et de la société hongroises se multiplient ; elles se différencient selon leur appartenance politique et économique ; mais leur trait commun est d'attribuer un rôle décisif aux petites et moyennes entreprises dans le processus de restructuration de l'économie et dans la création des nouveaux rapports de propriété.

Les données sur le développement économique basé sur les petites et moyennes entreprises - et en général pour la création de nouveaux rapports de marché - sont bien différentes selon les pays d'Europe de l'Est et d'Europe centrale. L'attraction pour un développement à la façon de l'Europe de l'Ouest s'explique par les spécificités d'évolution historique des divers pays de l'Europe centrale. La situation de la Hongrie à l'intérieur du bloc de l'Europe de l'Est est particulière en raisons des effets disparates des réformes économiques qui ont été lancées il y a deux décennies et demi. Les expériences hongroises de semimarché facilitent - sans aucun doute - la transition à l'économie de marché malgré le manque de capital, le besoin critique de modernisation ainsi que le développement politique d'alternatives adaptées au circonstances. Mais l'évolution rapide du secteur des PME pousse l'économie hongroise vers la décentralisation économique, régionale et politique.

\section{La formation du système des entreprises}

On peut déceler une dichotomie particulière entre la grande industrie et la petite production dans l'histoire des dernières cent années de l'économie hongroise 
moderne. À la fin du $\mathrm{xIx}^{\mathrm{e}}$ siècle, l'industrie manufacturière a pris un essor rapide en partant de presque rien. Ce processus d'industrialisation a été couronné par le triomphe de la grande industrie mécanique. Alors qu'en 1880, les travailleurs de la grande industrie ne représentaient que $21 \%$ de toute la main-d'œuvre manufacturière, au tournant du siècle, ils comptaient pour $40 \%$; et au début des années 1910, plus de la moitié des 563000 travailleurs manufacturiers et presque les trois quarts de la production industrielle appartenaient à la grande industrie. L'industrie manufacturière se caractérise par une concentration particulière ; on trouve plus du tiers des employés dans $4 \%$ des usines. Plus de la moitié des travailleurs manufacturiers travaille dans des usines de 21 à 100 employés ou dans celles engageant plus de mille personnes. La catégorie des entreprises moyennes n'existe presque pas. La concentration dans quelques branches de l'industrie lourde et la faiblesse des autres secteurs fabriquant des produits de consommation ont joué un grand rôle dans le processus de formation de la structure manufacturière.

La nouvelle situation après la Première Guerre mondiale - le territoire national a diminué des deux tiers et la population de $60 \%$ - n'a pas changé beaucoup le niveau de concentration du début du siècle. Mais à cette époque les usines de taille moyenne - qui étaient à peu près inexistantes au début du siècle - se sont multipliées grâce au développement de l'industrie du textile et des pâtes et papiers, etc. Les petites entreprises ont continué à employer $45 \%$ des travailleurs industriels et, en 1930, elles fournissaient $28 \%$ de la production industrielle ; $70 \%$ de la production des petites entreprises provenait de l'industrie de l'habillement, de l'alimentation, du bâtiment et de l'hôtellerie.

Le développement de l'industrie de guerre en vue de la Deuxième Guerre mondiale a touché non seulement les grandes mais aussi les petites entreprises. La production de guerre a renforcé économiquement la petite entreprise, bien que sa part de la production manufacturière ait chuté à $25 \%$ en 1940 .

En 1945, la production de l'industrie manufacturière se situa au cinquième niveau d'avant-guerre ; en 1946, elle dépassa à peine le tiers de celle-ci ; mais en 1948, elle atteignait déjà le niveau de production de la dernière année de paix. Et même au cours de cette année, on avait commencé à changer fondamentalement les rapports de propriété et le système d'organisation de l'économie hongroise. Cette transformation qui dura deux ans scella le sort du développement de l'économie hongroise ; durant quatre décennies, elle a coupé celle-ci des courants économiques modernes de l'Europe.

La construction de l'économie socialiste commença avec la loi XXV de 1948, qui instaura la nationalisation de toutes les entreprises hongroises manufacturières, minières, métallurgiques, de transports et de production électrique où l'effectif ouvrier dépassait 100 personnes à l'entrée en vigueur de la 
loi. Cette nationalisation toucha 600 entreprises ; 83,5\% des employés de toute l'industrie manufacturière passèrent au secteur public. Presque toutes les entreprises de l'industrie lourde furent nationalisées et seuls $25 \%$ des employés de l'industrie textile, $13 \%$ de ceux de la tannerie, $55 \%$ de ceux de l'industrie alimentaire restèrent dans le cadre des entreprises privées. Puis à la fin de l'année 1949 , les entreprises industrielles employant plus de 10 personnes furent nationalisées, et même, certaines branches industrielles passèrent entièrement au domaine public. Les deux tiers du commerce extérieur, le commerce de gros en entier et une grande partie du commerce de détail furent nationalisés.

Parmi les changements majeurs dans l'organisation de l'économie, on assista à une augmentation très forte de la concentration de la production. Ainsi, en 1950 le nombre des entreprises manufacturières nationalisées était de 1427, en 1965, ce nombre était réduit à 839. Dans les années 1950, l'effectif moyen par entreprise passa de 336 à 1183 personnes. En 1950, les entreprises industrielles ayant moins de 500 employés engageaient $30 \%$ des ouvriers, alors que ce pourcentage en 1965 atteignait à peine $10 \%$. En même temps, les plus grandes usines de 5000 employés et plus occupaient $35 \%$ de toute la force ouvrière contre $17 \%$ pour la période précédente. Mais cette concentration extraordinaire de la main-d'œuvre - qui s'est accompagnée d'une concentration urbaine puisqu'à la fin des années 1960, déjà $44 \%$ des employés de l'industrie manufacturière travaillaient dans l'agglomération de Budapest - n'était qu'apparente ; en grande partie, ce sont les changements organisationnels, soit les mesures prises pour soutenir la centralisation, qui l'ont causée et non les vrais processus de concentration. Ainsi malgré cette concentration des entreprises, la production a continué à se faire en bonne partie selon les anciens modes de production, alors que la centralisation trop forte des entreprises a restreint la coopération entre les entreprises en produisant des situations de monopole désavantageuses et en empêchant les entreprises de s'adapter aux besoins des consommateurs.

La réforme économique lancée en 1968, la liquidation partielle du système de planification centrale et une certaine libéralisation des entreprises stoppèrent la croissance de la taille des usines et des entreprises et déclenchèrent même un processus inverse. À vrai dire, au cours du deuxième tiers des années 70 - sous la pression des cercles staliniens de l'État-parti - une nouvelle tendance au gigantisme recommença à apparaître dans l'industrie de transformation ; mais à la fin de ces années, la taille des entreprises se mit à nouveau à diminuer et continua progressivement avec l'application de plus en plus poussée de la réforme économique en Hongrie. Les mesures prises par le gouvernement permirent le démantèlement des blocs monopolitiques les plus évidents et les moins justifiables. Au début, cette décentralisation ne concerna que l'industrie alimentaire, mais après elle s'étendit à d'autres secteurs industriels. Cette modernisa- 
tion de la structure des entreprises s'accompagna de plus en plus d'actions à long terme et de la fin des traditions conservatrices pour progressivement s'aligner sur l'économie de marché. Ces mesures de décentralisation prises par le gouvernement reflétaient cependant les points de vue des entreprises : elles ne favorisaient guère l'autonomie de gestion et le développement de la compétence entrepreneuriale.

À cette époque, à la base de l'économie hongroise et dans la société civile, le travail socialement non organisé (c'est-à-dire la seconde économie) commença à prendre de l'extension. C'est le modèle agraire hongrois qui joua le rôle primordial dans l'essor de ce processus. Le réseau coopératif de l'agriculture hongroise s'est fondamentalement distingué du système de type « kolkhoze » de l'Europe de l'Est. En effet, en Hongrie il s'est développé une division particulière du travail entre les grandes usines agro-alimentaires et l'exploitation personnelle auxiliaire effectuée sur des terrains privés ou loués, bien que cette situation ait été régulièrement critiquée du point de vue politico-idéologique. Au début des années 80, un million d'exploitations personnelles et auxiliaires fournissaient $70 \%$ de la culture maraîchère, $51 \%$ de la production fruitière et assuraient $21 \%$ de l'élevage bovin, $51 \%$ de celui du porc et $40 \%$ de l'élevage de la volaille. Plus de la moitié des petits producteurs étaient associés aux usines agro-alimentaires, les deux tiers de toute la production du secteur des petits producteurs étaient directement acheminés aux consommateurs et aux ré-exploiteurs (Zoltan, 1985).

Le rôle de cette seconde économie dans le relèvement du niveau de vie et de la demande devint évident dans d'autres secteurs de l'économie. Ainsi, à la fin des années 70, les entreprises socialistes ne satisfont que $30 \%$ de la plupart des services de répartition. Cette seconde économie s'adapta rapidement aux besoins réels et en comparant celle-ci avec les tendances internationales, lors de la révision de la politique économique, les mesures administratives contre la seconde économie furent vite rayées de l'ordre du jour. La réforme permit l'expansion des besoins réels et ceux-ci permirent la consolidation du secteur informel.

\section{Les entreprises au seuil du changement de régime}

Avec ces activités économiques multiples effectuées en dehors des organisations socialistes, l'entreprise privée relevait donc de certaines traditions, au moins en germes qui pouvaient être développés avec des cadres juridiques convenables. Avec la création un peu précitée de ces cadres pour des formes collectives des 
petites entreprises, la direction politique de l'époque voulut surtout créer une soupape pour atténuer les tensions sociales prévisibles, en donnant la possibilité à presque toutes les couches importantes de la société de contrebalancer la diminution inévitable du niveau de vie avec une certaine forme d'entreprise informelle ou d'un second emploi. Mais, l'État n'eut ni le temps, ni l'énergie pour soutenir théoriquement cette réforme et prévoir l'ampleur des conséquences.

Le lot de décrets lancés à l'automne de 1981 créa un statut de type collectif de toutes sortes d'entreprises dont le fonctionnement fut libre à dater du $1^{\text {er }}$ janvier 1982, soit :

- Les collectivités économiques de travail. Celles-ci pouvaient être créées avec la participation d'au moins 2 et d'au plus 30 personnes après l'immatriculation du nom commercial après avoir obtenu la permission du conseil municipal. Les membres qui bénéficiaient de l'assurance sociale pouvaient trouver ainsi un emploi principal, ou même un emploi supplémentaire. Ces firmes pouvaient engager des employés, mais ceux-ci ne pouvaient dépasser la moitié du nombre des membres. Ces collectivités avaient à payer un impôt social de $3 \%$.

- Les collectivités économiques de travail d'entreprise. Celles-ci étaient assujetties aux mêmes règlements que les collectivités économiques de travail auxquels s'ajoutaient la garantie en argent liquide de l'entreprise.

- Les petites coopératives. Ces dernières pouvaient être créées comme des entreprises organisées par les citoyens, sur une base volontaire et avec la participation d'au moins 10 et d'au plus 100 personnes. Par rapport aux coopératives traditionnelles, elles bénéficiaient des avantages de réglementation, comme l'exemption de la réglementation de salaire.

- Les groupements spécialisés de travaux industriels et de services de la coopérative. Pour créer ces groupements, il suffisait d'avoir l'accord de l'assemblée générale de la coopérative. Ce type d'entreprise devait avoir au moins cinq membres ; par contre, il n'y avait pas de limites d'employés. Ils étaient appelés avant tout à aider - par des activités industrielles plus rentables - les coopératives agricoles à améliorer leur organisation susceptible d'être à la source des rendements médiocres.

Pour le secteur des entreprises, outre les collectivités économiques de travail d'entreprise, on permit la création de filiales et de petites entreprises en premier lieu pour que les structures surcentralisées soient contrebalancées. Bien qu'elles aient bénéficié d'avantages par rapport entre autres à la réglementation salariale, et aux impôts, elles ne réussirent pas à se généraliser à cause du manque de motivation véritable des grandes entreprises. 
Tout bien considéré, on peut dire que ces types collectifs d'entreprises apportèrent des changements remarquables par rapport aux modèles staliniens de l'économie des pays de l'Europe de l'Est. En même temps, la "désétatisation » ne connut pas de grand succès en Hongrie pour les raisons suivantes :

- la pression politico-idéologique de la gauche conservatrice s'est maintenue jusqu'en 1989 au détriment des petites entreprises ; celles-ci continuèrent de subir de nombreuses restrictions administratives, et ne reçurent que peu de vrai soutien des politiques économiques.

- les privilèges de la propriété d'État ne diminuèrent pas considérablement ; l'effet paralysant de la propriété collective s'étendit même aux formes de propriété non étatiques.

La nouvelle période d'essor des entreprises en Hongrie date de 1988 lorsqu'on liquida les obstacles inhérents à la Constitution et vota les lois concernant le secteur privé. Ainsi, la loi VI de 1988 sur les collectivités a ouvert la voie au développement du secteur privé en Hongrie. Cette loi :

- permit aux petites entreprises privées de s'agrandir en faisant passer le nombre d'employés possibles de 30 à 500 personnes ;

- donna la possibilité à une même personne du secteur privé de créer plus d'une entreprise ;

- introduisit l'institution de sociétés à responsabilité limitée et de sociétés anonymes, dans lesquelles la responsabilité du propriétaire se limite à son apport ;

- légalisa l'institution de commanditaire; il devint ainsi possible de s'associer pour le capital en exclusivité.

La loi de 1988 sur les investissements des étrangers facilita remarquablement les investissements étrangers en Hongrie. Elle supprima le système précédent de concession, rendit possible la propriété à $100 \%$ par des étrangers et assura divers types d'exemptions d'impôt pour les sociétés à propriété mixte.

La loi dite de transformation entrée en vigueur au milieu de l'année 1989 permit à une partie des petites entreprises de choisir une forme de société plus conforme à leurs besoins, et résolut les problèmes de croissance et du changement de structure des petites entreprises. Par exemple, les collectivités économiques de travail purent se transformer de façon flexible, rapidement et gratuitement sans devoir au préalable être liquidées et verser un impôt ou une taxe spéciale. 
La loi sur l'entreprise privée de 1990 supprima la plupart des restrictions relatives à la petite industrie et au commerce privé et déclara valable le principe d'entreprise d'activité : ce n'est pas au type d'entreprise mais à l'activité qu'elle lia les arrêtés d'autorisation de l'autorité et du brevet.

Enfin, la loi de 1990 sur la Bourse et sur l'émission de titre supprima le minimum de valeur de départ des actions et rendit possible la participation des personnes privées à la Bourse de Budapest.

Bien que ces changements juridiques favorables n'aient pas été accompagnés d'une diminution acceptable des taxes d'État, une nouvelle vague d'entreprises se développa rapidement en Hongrie. Le nombre de celles-ci doubla presque entre les premiers trimestres de 1989 et de 1990. Ce sont les sociétés à responsabilité limitée qui se développèrent le plus dynamiquement, leur nombre ayant quadruplé.

Une des caractéristiques de ce dynamisme est que $42 \%$ des entreprises fonctionnant en 1990 furent créées entre 1989 et $1990 ; 32,1 \%$ avaient de 2 à 5 ans et seulement $25,9 \%$ existaient 5 ans auparavant. Les petites entreprises utilisant une comptabilité simplifiée en partie double et ayant un revenu annuel atteignant entre 250000 et $250000000 \mathrm{Ft}$ (entre 300000 et 3 millions de dollars) - eurent un rôle décisif à jouer dans la montée en flèche de ces entreprises.

À la fin de 1990, plus des trois quarts des entreprises étaient des petites organisations. Dans les domaines matériels, une petite organisation employait en moyenne 23 personnes avec des actifs de 9 millions Ft (120 000 \$ US), et des profits moyens de 28 millions Ft (380 000 \$ US). L'importance croissante des petites entreprises peut être vue dans le fait qu'à la fin de l'année 1990, dans l'industrie manufacturière, $45 \%$ des entreprises employaient moins de 20 personnes, alors que dans l'industrie du bâtiment, ce pourcentage était de $55 \%$, de $88 \%$ dans le commerce extérieur et de $77 \%$, dans le commerce intérieur. Les deux tiers des organisations industrielles avaient moins de 50 employés.

Ainsi, l'économie hongroise a changée rapidement son système politique et économique avec une structure industrielle où les PME sont devenues très importantes. 
TABLEAU 1

Statistique des établissements, 1985-1990

Organisation gérant

Organisation fonctionnant selon le type d'entreprise

- Coopérative

- Entreprise et trust

- Société à responsabilité limitée

- Société anonyne

- Entreprises associatives

- Entrepreneurs individuels
Le nombre d'établissement

\begin{tabular}{|c|c|c|c|c|c|c|c|}
\hline 1985 & 1986 & 1987 & 1988 & 1989 & 1990 & 1985 & 1989 \\
\hline 7,916 & 8,578 & 9,597 & 10,811 & 15,235 & 29,470 & 372,3 & 193,6 \\
4,848 & & & 6,880 & 7,076 & 7,132 & 147,1 & 114,9 \\
1,910 & 1,940 & 1,955 & 1,986 & 2,001 & 2,363 & 12,7 & 118,1 \\
50 & 59 & 103 & 451 & 4,485 & 18,307 & 366 fois + & 408,4 \\
& & & 116 & 307 & 646 & & 210,4 \\
17,849 & 20,262 & 22,674 & 34,082 & 44,062 & 65,400 & 366,4 & 148,4 \\
126,175 & 149,544 & 201,348 & 290,877 & 320,619 & 393,450 & 311,8 & 122,7 \\
\hline 151,940 & $\mathbf{1 7 8 , 3 8 4}$ & $\mathbf{2 3 3 , 6 1 9}$ & $\mathbf{3 3 5 , 7 7 0}$ & $\mathbf{3 7 9 , 9 1 6}$ & $\mathbf{4 8 8 , 3 2 0}$ & $\mathbf{3 2 1 , 4}$ & $\mathbf{1 2 8 , 5}$ \\
\hline
\end{tabular}

Source : Gazdaságstatisztikai Évkönyv, 1990, pp. 32-33 (Annuaire statistique d'économie). 
Mais malgré la libéralisation de l'économie, le développement d'un milieu économique plus favorable à l'économie privée et la transition à l'économie de marché, de même que le renforcement du secteur privé des petites et des grandes entreprises, la désintégration des entreprises étatiques a été très lente. La croissance de l'économie s'est fait attendre, car le profit croissant des nouvelles firmes a été contrebalancé par les pertes importantes et les faillites des entreprises traditionnelles, lesquelles augmentèrent pour diverses raisons : la transition à l'économie de marché, les contraintes dans l'intégration à l'économie de l'Ouest, la dévalorisation des usines pilotes de l'économie publique, la dévaluation des grandes firmes étatiques et l'ébranlement de la grande production agricole. Les infrastructures laissées à l'abandon ont entravé l'expansion de nouvelles entreprises en nécessitant de nouvelles liaisons d'information et de communication.

\section{La localisation et l'expansion territoriale des entreprises}

Dans la première section de cette analyse, nous nous sommes attardés au fait qu'aux éléments différenciant auparavant la structure territoriale de l'économie hongroise (la structure industrielle de l'économie, le niveau, l'exportation, les liaisons hiérarchiques, le développement technologique, etc.) s'ajoutent la forme de propriété et le niveau d'exploitation. Ainsi on pouvait observer, au milieu des années 80, les disparités d'économie entre la métropole et la province : $50 \%$ des entrepreneurs étaient installés dans la capitale avec $19,4 \%$ de la population, alors que $21 \%$ des employés dans les branches industrielles se retrouvaient à Budapest.

À la fin des années 1980 , cette concentration territoriale commença à ralentir ; mais même en 1990, presque la moitié des entreprises en croissance rapide se trouvait à Budapest et $10 \%$ de celles-ci était dans le district de Pest. Ce n'est que dans le cas des firmes privées (en raison de l'existence en province d'un grand nombre d'exploitations agricoles) que la capitale n'a que sa part démographique, comme on peut le voir au tableau 2.

De plus, si l'on examine le rôle de Budapest et de son agglomération relativement à la densité des organisations, on remarque la même situation. Par rapport au nombre d'entreprises par 10000 habitants, la métropole en compte 46,4 et l'agglomération, 18 - la moyenne nationale est de 18,7 . 
TABleaU 2

La répartition territoriale des entreprises

\begin{tabular}{|c|c|c|c|c|c|}
\hline Département & $\begin{array}{c}\text { Entreprise } \\
\text { coopé- } \\
\text { rative }\end{array}$ & $\begin{array}{c}\text { Société } \\
\text { économique }\end{array}$ & $\begin{array}{c}\text { Entreprise } \\
\text { de person- } \\
\text { nalité } \\
\text { non } \\
\text { juridique }\end{array}$ & $\begin{array}{c}\text { Entreprise } \\
\text { individuelle }\end{array}$ & Total \\
\hline Budapest & 32,4 & 48,2 & 32,6 & 22,7 & 24,1 \\
\hline Baranya & 4,7 & 2,7 & 3,2 & 5,2 & 4,9 \\
\hline Bács-Kiskun & 4,4 & 3,7 & 4,7 & 5,4 & 5,3 \\
\hline Borsod-Abauj-Zemplén & 5,3 & 3,8 & 5,8 & 5,2 & 5,3 \\
\hline Csongrád & 3,6 & 3,2 & 3,7 & 5,2 & 5,0 \\
\hline Fejér & 3,2 & 2,7 & 3,2 & 4,6 & 4,5 \\
\hline Gyõr-Moson-Sopron & 4,5 & 3,2 & 3,8 & 4,5 & 4,4 \\
\hline Hajdu-Bihar & 3,3 & 2,6 & 3,6 & 4,6 & 4,5 \\
\hline Heves & 2,4 & 1,9 & 2,8 & 2,9 & 3,0 \\
\hline Jász-Nagykun-Szolnok & 2,7 & 1,7 & 2,5 & 3,7 & 3,4 \\
\hline Komáron-Esztergom & 2,3 & 2,9 & 2,8 & 3,2 & 3,3 \\
\hline Nógrád & 2,0 & 1,3 & 2,1 & 1,9 & 1,9 \\
\hline Pest & 6,7 & 8,8 & 8,5 & 10,3 & 10,0 \\
\hline Somogy & 3,6 & 2,3 & 3,0 & 6,7 & 6,3 \\
\hline Szabolcs-Szatmár-Bereg & 3,6 & 2,4 & 4,4 & 4,4 & 4,5 \\
\hline Tolna & 2,1 & 1,4 & 2,6 & 0,9 & 1,0 \\
\hline Vas & 3,6 & 1,5 & 1,8 & 0,8 & 0,9 \\
\hline Veszprém & 3,7 & 2,1 & 3,4 & 2,1 & 2,2 \\
\hline Zala & 2,8 & 1,5 & 3,1 & 1,8 & 1,8 \\
\hline $\mathrm{Au}$ total & 100,0 & 100,0 & 100,0 & 100,0 & 100,0 \\
\hline
\end{tabular}

Source : Gazdaságstatisztikai Évkönyv 1990, pp. 32-35. Calculs de l'auteur 
On peut observer aussi que l'expansion lente des firmes privées, en comparaison avec le centre du pays, s'est surtout faite dans les départements voisins de la région de l'Ouest. Des indications montrent pourtant que la direction principale de cette expansion s'est faite dans l'axe économique de Vienne-GyörBudapest, d'une part, et dans l'axe de Trieste-Balaton-Budapest, d'autre part ; les régions du sud et du centre du pays n'ont été touchées que dans une faible mesure. Dans les régions périphériques de l'est et du nord-est, la densité demeure très faible. Ainsi, dans les départements les plus sous-développés, la valeur de l'indice est de 8,4 dans les zones de Szabolcs-Szatmar-Bereg ; de 7,7, dans celles de Jàsz-Nagykun-Szolnok ; de 9,7, dans le château fort de la grande industrie du département de Borsod-Abauj-Zemplén.

La situation géopolitique est plus favorable dans les départements situés à l'ouest du Danube qui, au milieu des années 80 , se sont rattachés à la dynamique régionale des régions économiques européennes des Alpes et de l'Adriatique ; les entreprises mixtes étrangères se sont alors implantées dans les grandes et les moyennes villes de cette région, tandis que les régions de l'Est étaient frappées par la désintégration du marché du COMECOM.

La reconstruction économique régionale nous amène enfin à revenir sur certains problèmes laissés jusqu'ici à l'arrière-plan. D'abord, on ne peut que constater que l'économie de marché commence à s'étendre au niveau régional. Mais, en dehors de Budapest, les marchés de capitaux, de main-d'œuvre, de marchandise et d'information ne fonctionnent que de manière partielle ; les institutions provinciales (même dans 5 ou 6 grandes villes du pays) sont limitées.

Les tendances donnent à penser que dans certains départements de l'ouest du pays, à court terme, un processus régional de développement, de type d'Europe occidentale, fondé sur les petites et les moyennes entreprises et sur le transfert technologique, et favorable à la croissance du secteur tertiaire, est en train de s'implanter. Pour cela, il faudra une politique économique gouvernementale favorisant les entreprises, décentralisant le pouvoir et stimulant les initiatives régionales. Mais aujourd'hui encore, la philosophie économique et les comportements du pouvoir politique central s'opposent à ces conceptions.

Pour remplacer un modèle uniforme à travers tout le pays, la Hongrie a besoin de politiques régionales qui favorisent la diversification des marchés, permettent de développer un marché financier régional et local et qui mettent en pratique différents moyens pour stimuler la création d'entreprises. 


\section{Bibliographie}

Cséfalvy, Z. et A. Nikodémus (1991), «A tervtõl a piacig. Féluton az elrugaszkodás elõtt ? » Du plan au marché. À mi-chemin de l'envol - ?, Valóság, 10, p. 11-21.

Csillag I. et L. Lengyel (1985), «Vállalkosás, állam, társadalom », Entreprise, état, société ; Budapest, Közgazdasági és Jogi Könyvkiadó.

ENYedi, Gy. (1990), « New Basis for Regional and Urban Policies in East-Central Europe », cahier de recherche $n^{\circ} 9$, Pécs, Centre d'études régionales.

Gazdaságstatisztika Évkőnyv (1990), annuaire statistique de l'économie, Budapest, Központi Statisztikai Hivatala.

HoRvÁth, Gy. (1991), « Regions in Competition : Internationale Division of Labour and Regional Policy », Horváth Gy. ed., Regional Policy and Local Governments, Pécs, Centre d'études régionales, p. 11-24.

Horváth. Gy. et L. Hrubi (1992), « Restructuring and Regional Policy in Hungary », cahier de recherche $n^{\circ} 12$, Pécs, Centre d'études régionales.

HaLÁsz, I. et Gy. SzzPESI (1983), « A kisüzemi gazdálkodás uj formái », Les nouvelles formes de l'économie des petites entreprises, Budapest, Közgazdasági és Jogi Könyvkiadó.

Matolcsy, Gy. (1991), « A magyar tõkefelhalmozás », L’accumulation du capital hongrois, Valóság 1, p. 1-13.

Nemes Nagy J. et É. RutTKay (1991), «Rural and Urban Forms of Private enterprises in Hungary », HoRváth Gy. éd., Régional Policy and Local Governments, Pécs, Centre d'études régionales, p. 163-173.

PETó, M. (1991), «A szervezeti és tulajdonviszonyok változáasa és a külföldi befektetések », La transformation des rapports de propriété et d'organisation et les investissements étrangers, Statisztikai Szemle, 12, p. 975-986.

Svrkőzy, T. (1990), «A privatizácio dilemmvi egy jogász szemszögébõl », Les dilemmes de la privatisation du point de vue d'un juriste, Aula, 3, p. 13-30.

SZELÉNYI, I. (1990), « Polgárosodás Magyarországon : nemzeti tulajdon polgársvg és polárosodó értelmiség », L'embourgeoisement en Hongrie : la bourgeoisie à capital national et l'intelligentsia bourgeoise, Valóság 1, p. 29-41. 
FIGURE 1

Pourcentage des chômeurs par département

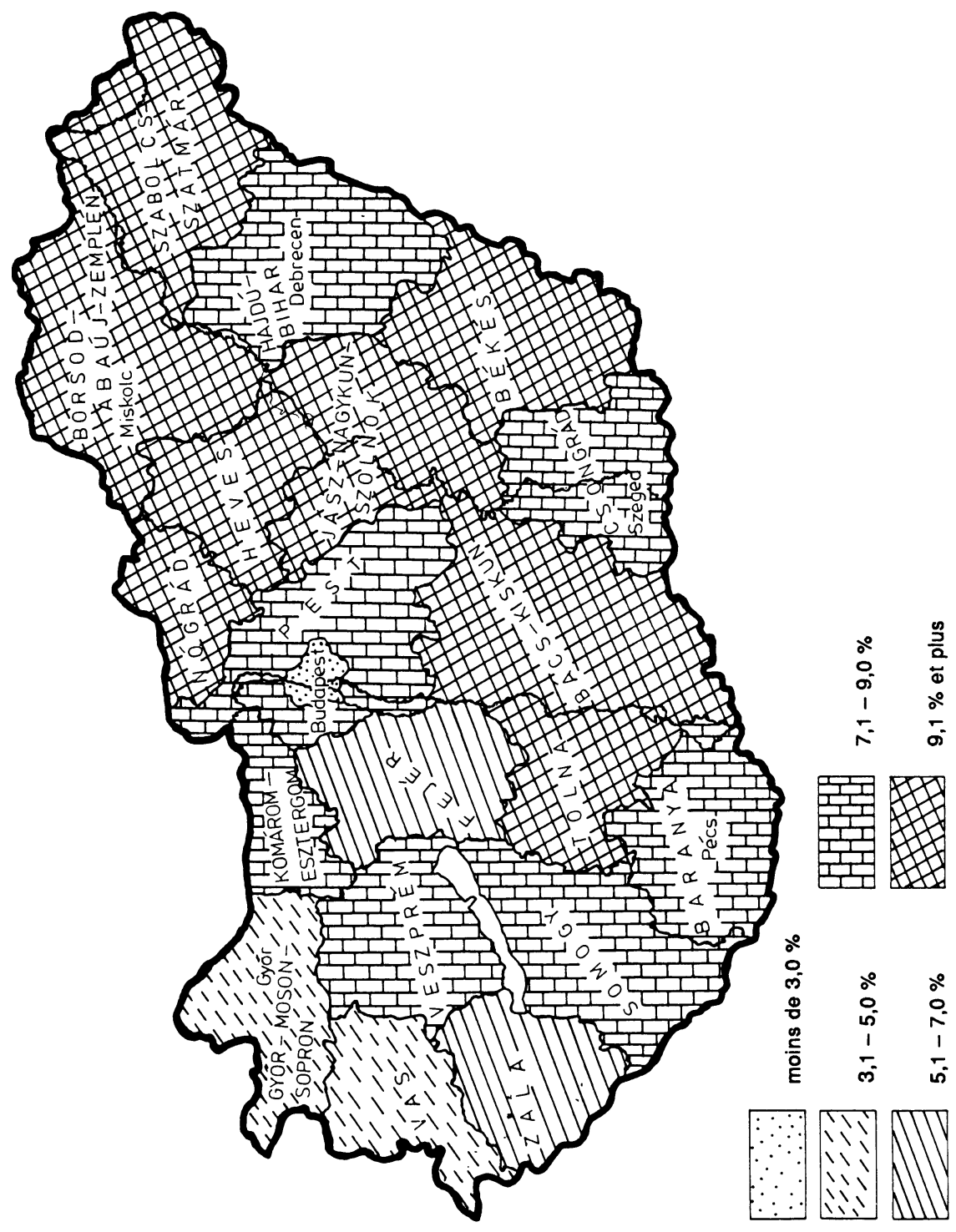


FIGURE 2

Répartition des entreprises en Hongrie

Signes explicatifs : nombre d'entreprises par 10000 habitants

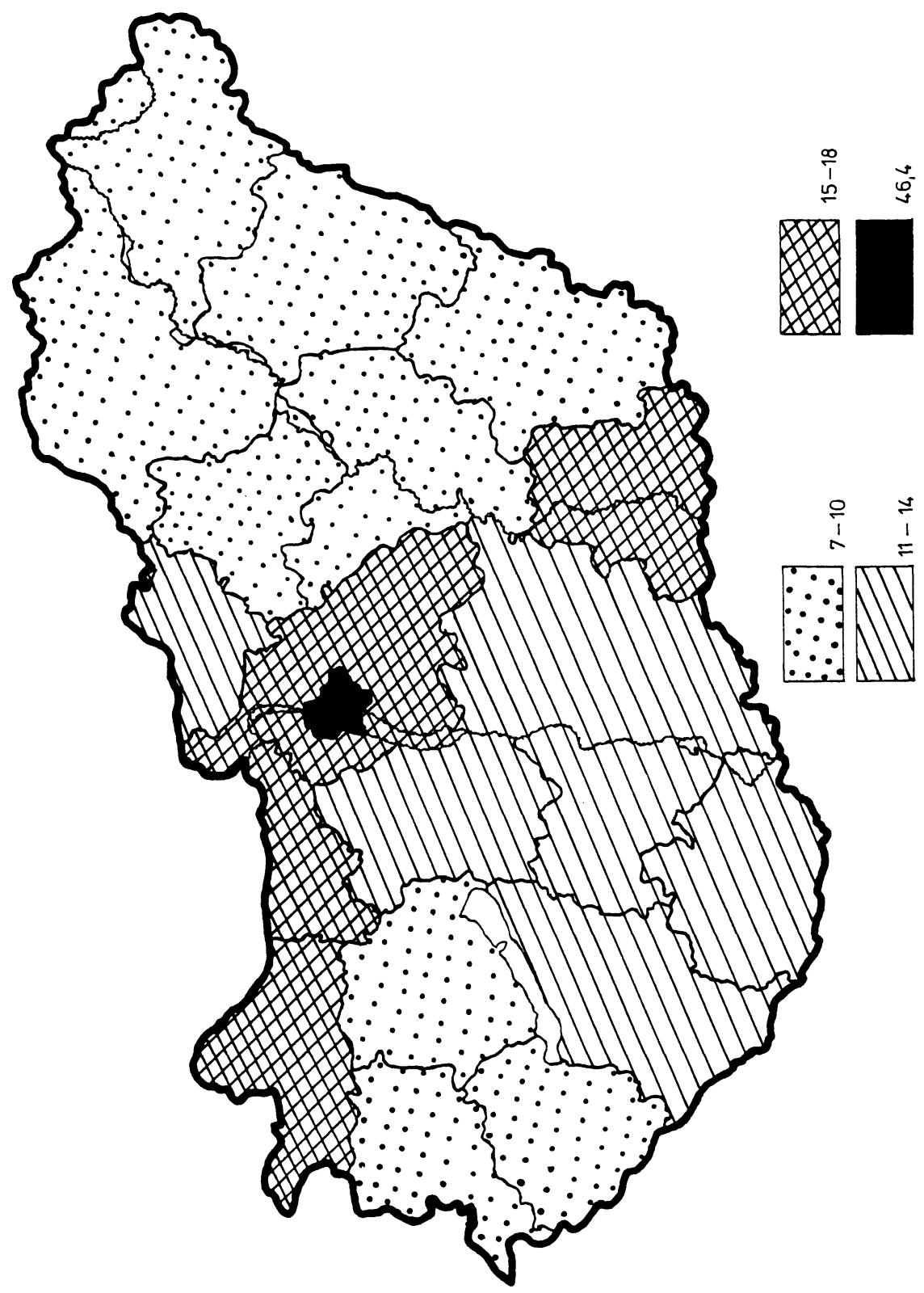

